\title{
Corrigendum
}

\section{A density-functional calculation of dynamic dipole polarizabilities of noble gas atoms}

\author{
S. K. Ghosh' ${ }^{1}$ and B. M. Deb ${ }^{2}$
}

${ }^{1}$ Heavy Water Division, Bhabha Atomic Research Center, Bombay 400085, India

${ }^{2}$ Department of Chemistry, Panjab University, Chandigarh 160014, India

Theoret. Chim. Acta (Berl.) 62, 209-217 (1983)

The $\Delta \bar{v}_{\text {eff }}^{1}$ terms in Eqs. (34) and (37) should be multiplied by a factor $1 / 2$. Table 1 should be replaced by the following table:

Table 1. Calculated values of dynamic (frequency-dependent) dipole polarizabilities of $\mathrm{Ne}, \mathrm{Ar}, \mathrm{Kr}$ and $\mathrm{Xe}$; all values are in atomic units ( 1 a.u. of polarizability $=4 \pi \varepsilon_{0} a_{0}^{3}=0.164878 \times 10^{-40} \mathrm{c}^{2} \mathrm{~m}^{2} \mathrm{~J}^{-1}$; 1 a.u. of frequency $=\hbar / m_{e} a_{0}^{2}=4.1341 \times 10^{16} \mathrm{rad} \mathrm{sec}^{-1}$ )

\begin{tabular}{|c|c|c|c|c|c|c|c|c|c|c|}
\hline \multirow[b]{2}{*}{$\begin{array}{l}\text { Frequency } \\
(\omega)\end{array}$} & \multicolumn{4}{|l|}{$\mathrm{Ne}$} & \multicolumn{2}{|l|}{$\mathrm{Ar}$} & \multicolumn{2}{|l|}{$\mathrm{Kr}$} & \multicolumn{2}{|l|}{$\mathrm{Xe}$} \\
\hline & $\begin{array}{l}\text { Present } \\
\text { work }\end{array}$ & $\begin{array}{l}\text { Ref. } \\
{[18]^{a}}\end{array}$ & $\begin{array}{l}\text { Ref. } \\
{[20]}\end{array}$ & $\begin{array}{l}\text { Ref. } \\
{[21]}\end{array}$ & $\begin{array}{l}\text { Present } \\
\text { work }\end{array}$ & $\begin{array}{l}\text { Ref. } \\
{[18]^{a}}\end{array}$ & $\begin{array}{l}\text { Present } \\
\text { work }\end{array}$ & $\begin{array}{l}\text { Ref. } \\
{[18]^{\mathrm{a}}}\end{array}$ & $\begin{array}{l}\text { Present } \\
\text { work }\end{array}$ & $\begin{array}{l}\text { Ref. } \\
{[18]^{\mathrm{a}}}\end{array}$ \\
\hline 0.00 & 4.89 & & 2.37 & 2.67 & 16.72 & & 19.36 & & 28.44 & \\
\hline 0.05 & 4.91 & & 2.37 & & 16.82 & & 19.46 & & 28.62 & \\
\hline 0.10 & 4.97 & & 2.39 & 2.69 & 17.14 & & 19.79 & & 29.20 & \\
\hline 0.15 & 5.08 & & 2.42 & & 17.72 & & 20.39 & & 30.26 & \\
\hline 0.20 & 5.26 & 2.80 & 2.46 & 2.77 & 18.69 & 12.51 & 21.37 & 19.68 & 32.04 & 35.15 \\
\hline 0.25 & 5.51 & 2.88 & 2.51 & & 20.30 & 13.50 & 22.95 & 21.87 & 35.07 & 44.20 \\
\hline 0.30 & 5.89 & 2.99 & 2.58 & 2.91 & 23.32 & 15.22 & 25.75 & 27.20 & 41.06 & 95.52 \\
\hline 0.35 & 6.48 & 3.14 & 2.68 & & 31.72 & 18.40 & 32.32 & & 62.42 & \\
\hline 0.40 & 7.56 & & 2.81 & 3.14 & & & & & & \\
\hline 0.45 & 10.60 & & 2.98 & & & & & & & \\
\hline
\end{tabular}

${ }^{a}$ Quoted in Ref. [19]

The first line in Table 2 should be replaced by the following line:

$\begin{array}{lllll}4.89 & 16.72 & 19.36 & 28.44 & \text { Present work }\end{array}$ 